\title{
Revista da Sociedade Brasileira de Medicina Tropical Volume 41(5): página 524
}

A Página 524 do Relatório Técnico "Consenso em criptococose - 2008” de autoria de Maria Luiza Moretti, Mariângela Ribeiro Resende, Márcia Dos Santos Lazéra, Arnaldo Lopes Colombo e Maria Aparecida Shikanai-Yasuda, foi publicada sem os autores principais e endereço para correspondência. Abaixo segue os dados faltantes da referida página.

\section{Consenso em criptococose -2008}

\author{
Guidelines in Cryptococcosis - 2008
}

Maria Luiza Moretti', Mariângela Ribeiro Resende, Márcia Dos Santos Lazéra², Arnaldo Lopes Colombo ${ }^{3}$ e Maria Aparecida Shikanai-Yasuda ${ }^{4}$

1. Departamento de Clínica Médica, Faculdade de Ciências Médicas, Universidade Estadual de Campinas, Campinas, SP. 2 Instituto de Pesquisa Clínica Evandro Chagas, Departamento de Parasitologia, Microbiologia e Imunologia; Fundação Oswaldo Cruz, Rio de Janeiro, RJ. 3. Departamento de Medicina, Escola Paulista de Medicina, São Paulo, SP. 4. Departamento de Moléstias Infecciosas e Parasitárias da Faculdade de Medicina da Universidade de São Paulo, São Paulo, SP.

Endereço para correspondência: Dra. Maria Luiza Moretti. Faculdade de Ciências Médicas/UNICAMP. Caixa Postal 6111, 13083-970 Campinas, SP. e-mail: moretti@hc.unicamp.br

Recebido para publicação em 15/08/2008

Aceito em 10/10/2008 